\title{
Recalibration of the Soft X-Ray Telescope Onboard Yohkoh
}

\author{
Loren W. Acton ${ }^{1}$
}

Received: 21 May 2018 / Accepted: 21 September 2018 / Published online: 5 October 2018

(C) The Author(s) 2018

\begin{abstract}
We present a new derivation of the X-ray spectral sensitivity of the Soft X-ray Telescope (SXT) experiment onboard Yohkoh. The recalibration is based upon the hypothesis that, during the first 15 months of the mission, an absorbing material gradually built up on the entrance filters of the telescope. We have also re-evaluated the times and sizes of ruptures of the SXT entrance filters. The impact of this recalibration on derived filter-ratio temperature, emission measure, and calculated spectral irradiance is substantial, especially for SXT data prior to November 1992.
\end{abstract}

Keywords Sun, X-rays · Corona, temperature $\cdot$ Solar irradiance $\cdot$ Instrumental effects

\section{Introduction}

The Soft X-ray Telescope (SXT) experiment on the Japan/US/UK Yohkoh mission recorded $\mathrm{X}$-ray images of the full Sun, active regions, and flares in the (nominal) $0.3-3.0 \mathrm{~nm}$ band for the period October 1991 to 14 December 2001. The solar observations by Yohkoh are archived in their entirety in the Yohkoh Legacy Archive (YLA) website (solar.physics.montana.edu/ylegacy/) and at ISAS/JAXA in Japan (darts.isas.jaxa.jp/solar/ yohkoh/) as well as the Solar Data Analysis Center of NASA Goddard Space Flight Center (umbra.nascom.nasa.gov/yohkoh/y4sdac_top.html). The latter archives provide less reduced data and related information than the YLA, which is described by Takeda et al. (2009), Takeda (2015), and Acton (2017). The Yohkoh mission is described in the collection of articles edited by Švestka and Uchida (1991). The individual articles are also available on the YLA.

The SXT instrument has been described by Tsuneta et al. (1991). On-orbit data issues and calibration are exhaustively treated by Acton (2016). There it is explained that the entrance apertures of the SXT were covered by duplex entrance filters. The individual filters

L.W. Acton

loren.acton@montana.edu

1 Department of Physics, Montana State University, P.O. Box 173840, Bozeman, MT 59717-3840, USA 
Figure 1 SXT entrance apertures with filter sectors numbered. The diameter of the telescope is $282.7 \mathrm{~mm}$.

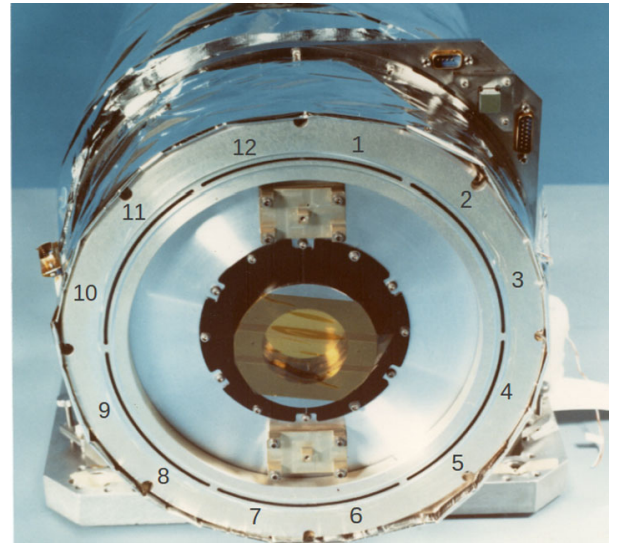

are mounted on frames each of which covers a 60 degree sector of the entrance annulus. Each frame comprises two individual 30 degree sections for a total of 12 sectors. The filter membrane is Lexan plastic $180 \mathrm{~nm}$ thick covered with $70 \mathrm{~nm}$ of Ti (to exclude $\mathrm{He}$ II, $30.4 \mathrm{~nm}$ ) and $90 \mathrm{~nm}$ of $\mathrm{Al}$ for thermal control and to exclude visible light. For the outer ring of filters the Al layer faces the Sun. The X-ray entrance filters encircle the visible-light aspect telescope as shown in Figure 1.

In the course of this re-examination of SXT calibration we have concluded that some absorbing material was deposited on the SXT aspect telescope entrance window and the outer X-ray entrance filters during the first $\approx 15$ months of the mission. For want of better knowledge we assume that this material is carbon (C), reaching a total thickness of about $0.225 \mu \mathrm{m}$ by the time (November 1992) that additional deposition became inconsequential.

Entrance-filter ruptures were detected from time to time up until 1999. We have reexamined the timing and magnitude of each entrance-filter failure. The results of this work are given in Table 2 .

The deposition of absorbing material and rupture of each entrance-filter sector changed the spectral sensitivity of the SXT. Thus, derivation of, e.g., temperature and emission measure from filter ratios must take these changes into account. The changes in SXT sensitivity were only approximately accounted for in previous calibrations (Acton, 2016). They are fully treated in the results presented in this article and in the updated SXT data analysis software archived in SolarSoft (sohowww.nascom.nasa.gov/solarsoft/).

\section{The Case for Entrance-Filter Contamination}

All elements of the SXT optical train were calibrated in the laboratory. End-to-end testing of the SXT under vacuum was consistent with the piecewise calibration results. SXT Calibration Notes 5 (Lemen and Hudson, 1990), 29 (Acton, 1992), and 30 (Lemen, 1992), which are available in the YLA, detail aspects of pre-launch calibration and testing. On-orbit issues affecting SXT calibration have been discussed by Acton (2016).

The signal from the SXT aspect telescope (usually referred to as the SXT aspect sensor) at $341 \mathrm{~nm}$ decreased exponentially immediately after launch (Figure 2), and had reached a level of about $8 \%$ by November 1992 . The physical reason for the decay remained a mystery for more than two decades. Deposition of some absorbing material on the entrance optic of the aspect sensor was rejected as the cause for two reasons. i) The black Chemglaze paint 

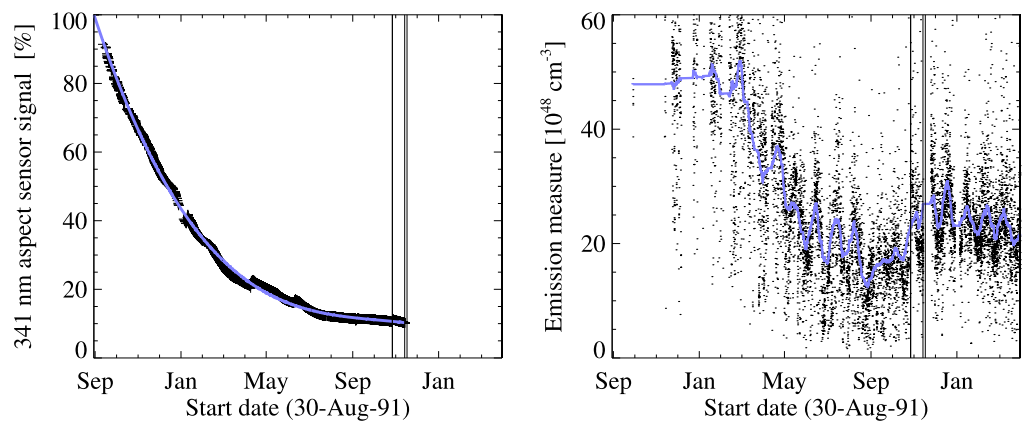

Figure 2 Left: decay of aspect-sensor signal, overlaid (blue line) with a third-order polynomial fit to the data. This curve is used to calculate the build up of $\mathrm{C}$ on the X-ray entrance filters. Right: full-Sun emission measure derived from 9510 filter-ratio pairs acquired prior to 1 April 1993. The blue line is a 301-point boxcar smoothing of the emission-measure results. Twenty-seven day modulation of the emission measures, from solar rotation, is evident. The vertical lines in both panels denote times of entrance-filter ruptures.

Table 1 X-ray transmission of an entrance-filter sector versus wavelength.

\begin{tabular}{llllll}
\hline $\begin{array}{l}\text { Wavelength } \\
{[\mathrm{nm}]}\end{array}$ & $\begin{array}{l}\text { Launch } \\
\text { Both filts. }\end{array}$ & $\begin{array}{l}\text { Launch } \\
\text { Inner only }\end{array}$ & $\begin{array}{l}\text { Oct 1992 } \\
\text { Both filts. }\end{array}$ & $\begin{array}{l}\text { Oct 1992 } \\
\text { Inner only }\end{array}$ & No filt. \\
\hline 0.50 & 0.89 & 0.95 & 0.89 & 0.95 & 1.00 \\
1.00 & 0.73 & 0.85 & 0.69 & 0.85 & 1.00 \\
1.50 & 0.41 & 0.64 & 0.34 & 0.64 & 1.00 \\
2.00 & 0.17 & 0.41 & 0.11 & 0.41 & 1.00 \\
2.50 & 0.08 & 0.29 & 0.04 & 0.29 & 1.00 \\
\hline
\end{tabular}

on the inner side the aluminum closeout plate immediately in front of the SXT had been chosen as an acceptable low-outgas material and had been prepared and baked out according to NASA-approved methods and no other probable source of contaminating material was identified. ii) X-ray images from the SXT revealed no obvious signature concurrent with the signal decline of the aspect sensor.

Recently, in the course of deriving soft X-ray irradiance for the entire Yohkoh mission, we noticed the strange temporal evolution of derived emission measure depicted in Figure 2. The steep fall-off of emission measure and its sharp increase at the times of the first entrancefilter failures suggest that the calibration prior to those ruptures employed an SXT effective area greater than was actually the case. This result would be experienced if some absorbing material accumulated on all front-end X-ray apertures in parallel with the contamination of the aspect-sensor entrance window. In such a scenario the amount of contaminant must be substantial to absorb over $90 \%$ of the $341 \mathrm{~nm}$ light falling on the aspect-sensor outer window. Such a layer of absorbing material accumulating on the X-ray entrance filters, covering the Al outer layer, would also severely change the thermal properties of these thin membranes, producing an enhanced thermal shock at every sunrise. (Note that, as far as we can tell, all entrance-filter failures took place at orbital sunrise.) Failure to take into account the diminishing X-ray sensitivity of the SXT as the contaminant built up would produce a temporal profile of emission measure similar to that shown in Figure 2. Looking ahead at results of this recalibration, values of SXT entrance-filter transmission, caused by deposition of $\mathrm{C}$ and subsequent failure of an outer entrance filter, are given in Table 1 where 
a comparison of columns 2 and 4 demonstrates the decreased X-ray transmission caused by absorption in the contamination layer.

\section{SXT Recalibration}

Here, recalibration refers to a revision of the transmission, i.e. effective area, of the entrance aperture of the SXT as a function of wavelength. The other factors (i.e. mirror reflectivity, transmission of analysis filters, CCD response) which are multiplied together with the entrance-filter effective area to determine the spectral response of the telescope are unchanged. While the decay of the aspect-sensor response is well determined, it is an assumption that the temporal profile of the build up of the (unknown) contaminant on the X-ray entrance filters matches that of the aspect sensor. The material accumulating on the SXT entrance apertures is assumed to be carbon (C), which has no absorption edges in the spectral band sampled by the SXT. If heavier elements with absorption edges below about 2.5 $\mathrm{nm}$ were present in the contaminant this would impact the spectral X-ray transmission of the contaminant and thus the calibration of the instrument. Even with these uncertainties we believe that the result of this recalibration is an improvement.

The only way to relate the X-ray absorption of the contaminant to the aspect-sensor decay curve is to find a situation for which the SXT observes the X-ray Sun immediately before and after outer-entrance-filter ruptures in order that solar X-ray intensity and spectrum are, as near as possible, the same. The before and after observations should be near together in time, because solar X-rays are constantly changing, and well away from particle contamination from the South Atlantic Anomaly. The outer entrance-filter failures of 27 October 1992, one orbit apart at 05:41 and 07:18, provide the best opportunity to determine the effective thickness of the hypothesized $\mathrm{C}$ layer at the time that entrance filters began to fail. This was a period of high flare activity, but we were fortunate to obtain thin- $\mathrm{Al}$ and $\mathrm{Al} / \mathrm{Mn} / \mathrm{Mg}$ filter pairs (see Acton, 2016 for descriptions of the SXT analysis filters) with comparable GOES flux levels before and after the two failures as illustrated in Figure 3.

We have no knowledge of what material accumulated on the SXT entrance apertures but, whatever it is, it is likely to include $\mathrm{C}$ in its composition. Unfortunately, the aspect-sensor
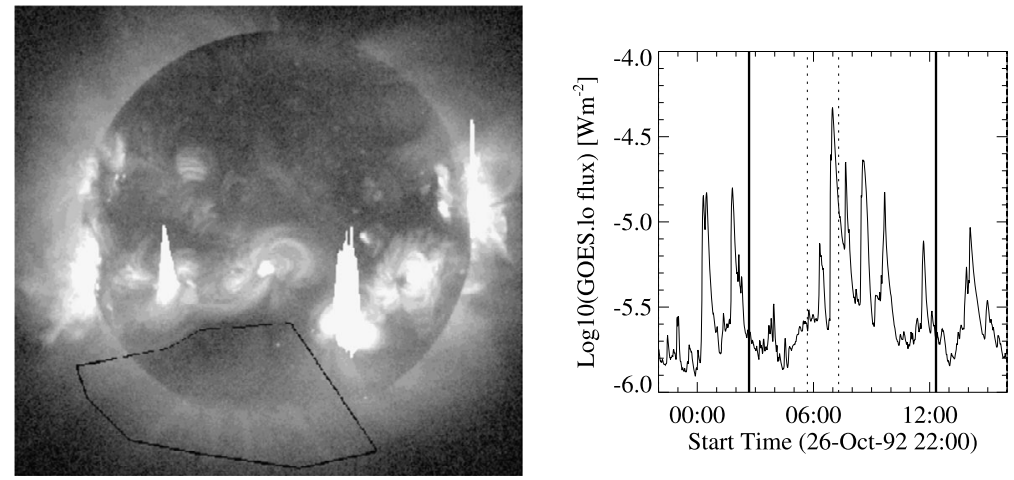

Figure 3 Left: SXT Al.1 image of 12:19:37 27 October 1992. The south polar region used for $\mathrm{C}_{\sigma}$ analysis is outlined by the black line. Right: times of SXT image pairs used for $\mathrm{C}$ thickness analysis compared to GOES low channel 1-8 ̊̊ flux. Vertical broken lines denote times of outer entrance-filter failures. Heavy vertical lines mark times of SXT image pairs. 

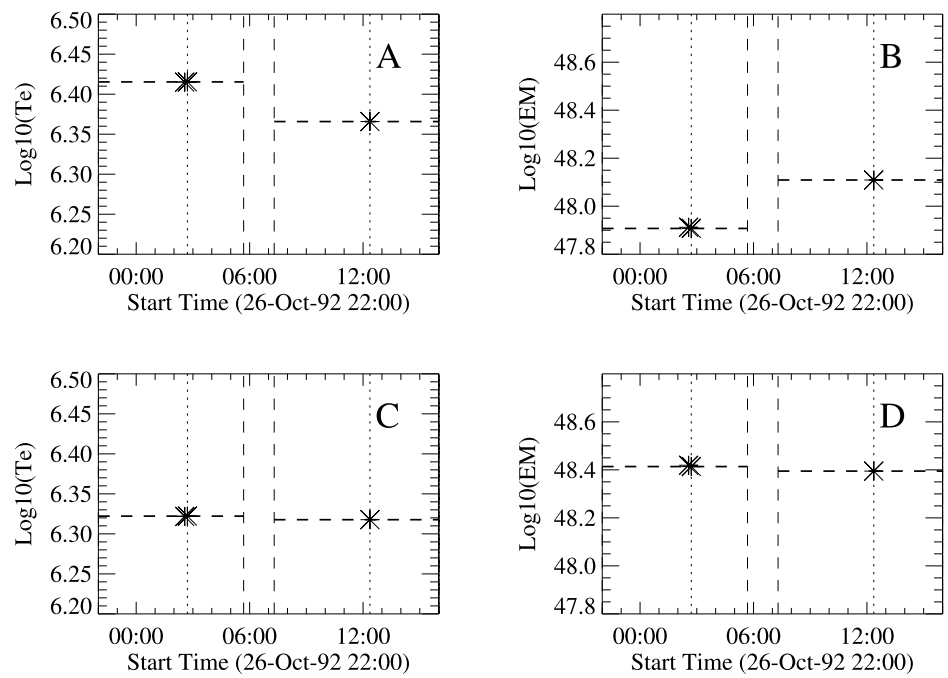

Figure 4 Panels $A$ and $B$ show the mismatch of coronal temperature and emission measure across the entrance failures for the launch calibration. Panels $C$ and $D$ are the corresponding transitions for a carbon deposition layer of $0.225 \mu \mathrm{m}$.

decay curve, while it provides the fractional rate of contaminant deposition, does not itself yield the thickness of the $\mathrm{C}$ layer. The linear absorption coefficient of $\mathrm{C}$ at $341 \mathrm{~nm}\left[\mathrm{C}_{\sigma}\right]$ appears to depend strongly upon deposition conditions and ranges from 0.75 (Dasgupta et al., 1991) to 2.3 (Palik, 1991) to 13 (Laidani et al., 2008) $\mu^{-1}$ in the literature. The absorption coefficient of $\mathrm{C}$ in $\mathrm{X}$-rays is well determined and readily available.

In order to avoid, as much as possible, variations in the coronal X-ray signal over this interval the region around the South Pole as delineated in Figure 3 was selected for analysis. It is convenient to parameterize the analysis of the $\mathrm{C}$ layer in terms of the (unknown) linear absorption coefficient $\left[\mathrm{C}_{\sigma}\right]$ at $341 \mathrm{~nm}$, the wavelength of the narrow-band aspect-sensor images. This readily converts to $\mathrm{C}$ thickness.

A perfect match across the filter failures requires $C_{\sigma}=9.6$ for temperature and $C_{\sigma}=10.7$ for emission measure. Thus, we have chosen $\mathrm{C}_{\sigma}=10.1$, corresponding to a $\mathrm{C}$ thickness of $0.225 \mu \mathrm{m}$. It is encouraging that the derived value of $\mathrm{C}_{\sigma}$ (i.e. 10.1) falls within the published range mentioned above. Figure 4 displays the result of the SXT entrance-filter recalibration including the $\mathrm{C}$ layer. A satisfactory match is achieved for both temperature and emission measure.

Acton (2016) discusses, in detail, the sequence of failures of the SXT entrance filters based primarily upon visible stray light within the telescope. In this work stray-light signatures in the corners, with little or no X-ray illumination, of the raw full-frame X-ray images have been included in the analysis in order to provide better temporal coverage and resolution. It was thus discovered that the November-1992 inner-filter ruptures took place in two steps, as shown in Figure 5. All failure events were examined in a similar manner. Table 2 presents the conclusions of this work. Note that, after October 1992, it is not possible to see stray-light effects from ruptures in outer entrance filters alone, so the table gives the same values for the number of open sectors for both outer and inner filters.

Figure 6 illustrates the improved match of physical parameters derived from SXT fullSun filter pairs across the time of the two outer-filter ruptures of 27 October 1992. Note that 


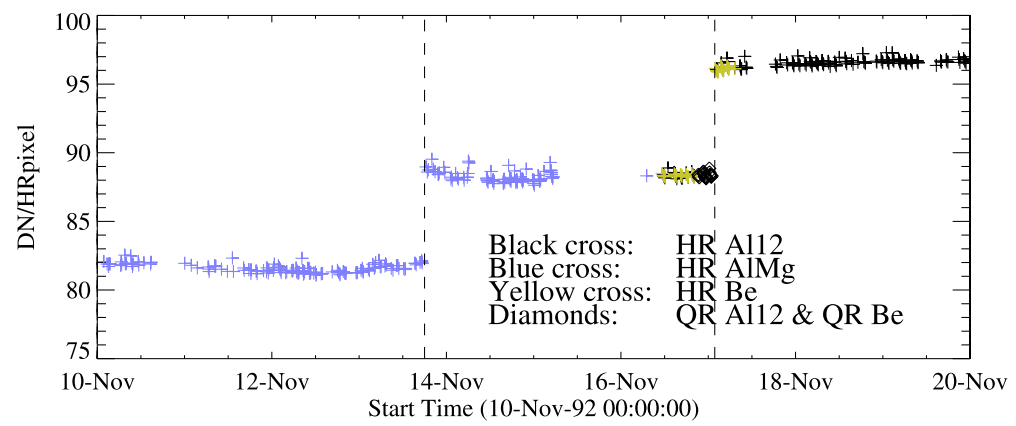

Figure 5 Signals from a small area in the lower-right corner of raw X-ray images for various resolution and analysis filters. The units given on the $y$-axis refer to the blue crosses. Other data have been adjusted to the half-resolution (HR) data taken through the AlMg filter. The steps are caused by increases in visible stray light reaching the focal plane at the times of entrance-filter ruptures.

Table 2 Revised sequence of SXT entrance-filter failures.

\begin{tabular}{lll}
\hline Date & $\begin{array}{l}\text { Outer } \\
\text { sectors }\end{array}$ & $\begin{array}{l}\text { Inner } \\
\text { sectors }\end{array}$ \\
\hline 30-Aug-91 10:30 & 0.0 & 0.0 \\
27-Oct-92 05:41 & 1.0 & 0.0 \\
27-Oct-92 07:18 & 2.0 & 0.0 \\
13-Nov-92 18:00 & 2.0 & 1.0 \\
17-Nov-92 01:47 & 2.0 & 2.0 \\
16-Aug-95 08:04 & 4.0 & 4.0 \\
24-Aug-96 07:00 & 5.0 & 5.0 \\
24-Jan-98 00:00 & 6.0 & 6.0 \\
30-Jan-99 23:17 & 8.0 & 7.0 \\
12-Mar-99 02:00 & 8.0 & 7.5 \\
20-Apr-99 19:02 & 8.0 & 8.0 \\
14-Dec-01 21:12 & 8.0 & 8.0 \\
\hline
\end{tabular}

coronal temperatures are decreased and emission measures and derived fluxes are increased by the new calibration.

\section{Conclusion}

The analysis of SXT data reported here support the hypothesis that some carbon-like material deposited on the entrance apertures of the SXT. The rate of deposition decreased to near zero by the end of 1992 and its impact on calibration became of less importance as more sectors of the entrance filters ruptured, removing the contaminated outer entrance filters from the optical path.

The impact, on temperature and X-ray irradiance, of the new calibration is illustrated in Figure 7. During the period up to the time of the first entrance-filter failure the derived filter-ratio temperature decreased, as compared to the old calibration, as the contamination increased in thickness. During this time, more than $60 \%$ of SXT measurements of the wholecorona temperature fell between 2 and $3 \mathrm{MK}$, producing the distinct curve corresponding to 

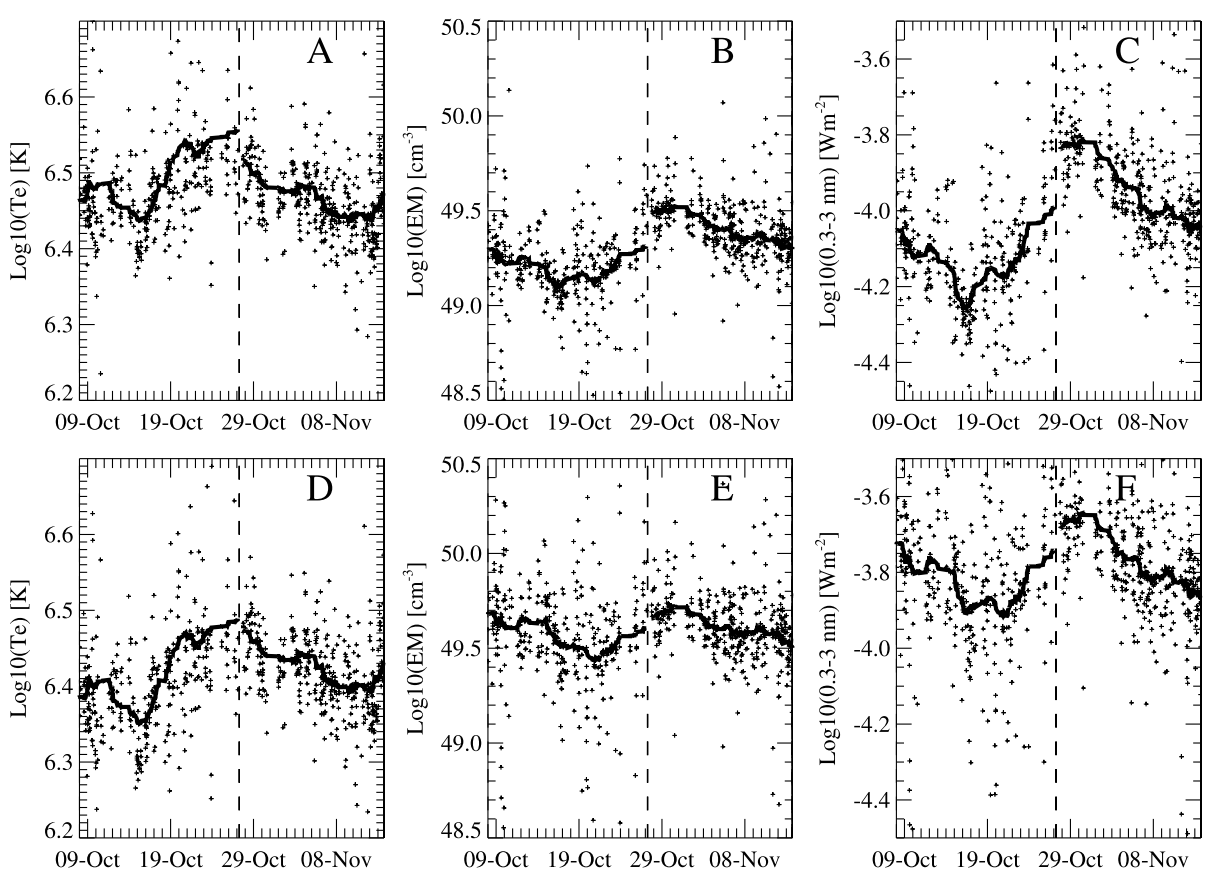

Figure 6 Panels $A, B$, and $C$ show the mismatch of the run of derived full-Sun coronal temperature, emission measure, and $0.3-3 \mathrm{~nm}$ irradiance across the first two entrance failures, indicated by the broken lines, for the old calibration. Panels $D, E$, and $F$ illustrate the improvement with the new SXT calibration with a carbon deposition layer reaching $0.225 \mu \mathrm{m}$ in thickness. The solid lines are generated with a 61-datum running boxcar smoothing before and after the broken line.
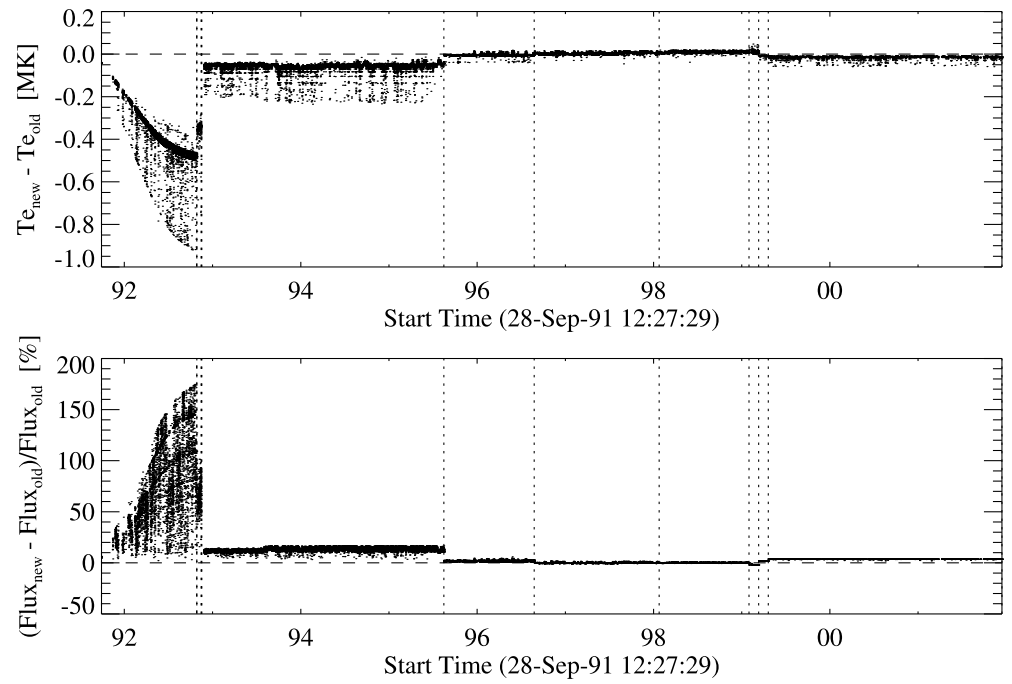

Figure 7 Top: difference in derived coronal temperature resulting from the new SXT calibration. Bottom: percentage difference in $0.3-3 \mathrm{~nm}$ irradiance resulting from use of the new calibration. 
this temperature range in the plot. The points below it correspond to higher derived temperatures, usually associated with flaring. The scatter in the lower, change-in-irradiance, curve reflects both the temperature dependence of computing irradiance and the temperature dependence of the emission measure derived from the SXT signal levels.

As demonstrated in Figure 7, published SXT analyses using the old calibration for post-November-1992 SXT observations, and especially for observations after August 1995, should not be seriously impacted by the recalibration reported herein.

Acknowledgments We sincerely thank the Physics Department of Montana State University for an Emeritus position and the use of the infrastructure necessary to do this research. Aki Takeda, creator of the YLA, has contributed to this work in many ways. This article capitalizes on the Yohkoh mission, software, and data products supported by NASA over several decades.

Disclosure of Potential Conflicts of Interest The author declares to have no conflicts of interest.

Open Access This article is distributed under the terms of the Creative Commons Attribution 4.0 International License (http://creativecommons.org/licenses/by/4.0/), which permits unrestricted use, distribution, and reproduction in any medium, provided you give appropriate credit to the original author(s) and the source, provide a link to the Creative Commons license, and indicate if changes were made.

\section{References}

Acton, L.: 1992, SXT Calibration Note 29. Preliminary Calibration of SXT Flight X-ray Filters. solar.physics. montana.edu/ylegacy/documents/sxt_cal_note/SXT_cal_note_29.pdf.

Acton, L.W.: 2016, On-orbit performance and calibration of the soft X-ray telescope on Yohkoh. Solar Phys. 291, 643. DOI. ADS.

Acton, L.W.: 2017, The Yohkoh legacy (data) archive. Phys. Astron. Int. J. 1(3), 00019. DOI.

Dasgupta, D., Demichelis, F., Pirri, C.F., Tagliaferro, A.: 1991, $\pi$ bands and gap states from optical absorption and electron-spin-resonance studies on amorphous carbon and amorphous hydrogenated carbon films. Phys. Rev. B 43, 2131. DOI. ADS.

Laidani, N., Bartali, R., Gottardi, G., Anderle, M., Cheyssac, P.: 2008, Optical absorption parameters of amorphous carbon films from Forouhi Bloomer and Tauc Lorentz models: a comparative study. J. Phys., Condens. Matter 20(1), 015216. DOI. ADS.

Lemen, J.R.: 1992, SXT Calibration Note 30. Effective Area of SXT Mirror (In-Flight). solar.physics. montana.edu/ylegacy/documents/sxt_cal_note/SXT_cal_note_30.pdf.

Lemen, J.R., Hudson, H.S.: 1990, SXT Calibration Note 5. The SXT X-ray Neutral-density Filters. solar. physics.montana.edu/ylegacy/documents/sxt_cal_note/SXT_cal_note_5.pdf.

Palik, E.D. (ed.): 1991, Handbook of Optical Constants of Solids II, Academic Press, Boston.

Švestka, Z., Uchida, Y. (eds.): 1991, The YOHKOH (SOLAR-A) Mission, Kluwer, Dordrecht.

Takeda, A.: 2015, Yohkoh Legacy Data Archive. solar.physics.montana.edu/ylegacy.

Takeda, A., Acton, L., McKenzie, D., Yoshimura, K., Freeland, S.: 2009, Resident archive services of the Yohkoh legacy data archive. Data Sci. J. 8, IGY1. DOI.

Tsuneta, S., Acton, L., Bruner, M., Lemen, J., Brown, W., Caravalho, R., Catura, R., Freeland, S., Jurcevich, B., Owens, J.: 1991, The soft X-ray telescope for the SOLAR-A mission. Solar Phys. 136, 37. DOI. ADS. 